\title{
Three-dimensional distribution of water and air in soil pores: Comparison of two-phase two-relaxation-times lattice-Boltzmann and morphological model outputs with synchrotron X-ray computed tomography data
}

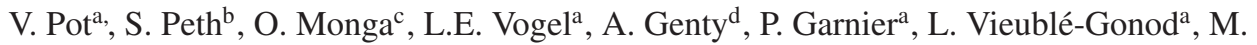 \\ Ogurreck $^{\mathrm{e}}$, F. Beckmann ${ }^{\mathrm{e}}$, P.C. Baveye $\mathrm{B}^{\mathrm{a}, \mathrm{f}}$ \\ ${ }^{a}$ INRA, AgroParisTech, UMR 1402 ECOSYS, F-78850 Thiverval-Grignon, France \\ ${ }^{b}$ Department of Soil Science, University of Kassel, Nordbahnhofstr. 1a, D-37231 Witzenhausen, Germany \\ ${ }^{c}$ UMMISCO-Cameroun, UMI 209 UMMISCO, University of Yaoundé, IRD, University of Paris 6, F-93143 Bondy \\ Cedex, France \\ ${ }^{d}$ CEA Saclay, DEN/DANS/DM2S/STMF/LATF, F-91191 Gif-sur-Yvette, France \\ ${ }^{e}$ GKSS-Research Centre, Max-Planck-Str. 1, D-21502 Geesthacht, Germany \\ ${ }^{f}$ Laboratory of Soil and Water Engineering, Rensselaer Polytechnic Institute, Troy, New York 12180, USA
}

\begin{abstract}
Recent progress in the understanding of soil microbial processes at micrometric scales has created a need for models that accurately predict the microscale distribution of water, and the location of air-water interfaces in pores. Various models have been developed and used for these purposes, but how well they fare against real data has yet largely to be determined. In this context, for the first time, this article compares the prediction of two of these models to experimental data obtained on soil material. The distribution of water and air in soil samples constituted of repacked aggregates, equilibrated at three matric potentials $(-0.5 \mathrm{kPa},-1 \mathrm{kPa}$ and $-2 \mathrm{kPa})$, was measured via synchrotron X-ray computed tomography at a resolution of $4.6 \mu \mathrm{m}$. Water distribution was simulated by a two-phase lattice Boltzmann model (LBM) and a morphological model (MOSAIC). Results indicate that, when one lifts the assumption, motivated by capillary theory, that a pore can drain only if a connecting pore is already full of air, MOSAIC gives an acceptable approximation of the observed air-water interfaces. However, discretization of pores as geometrical primitives causes interfaces predicted by MOSAIC to have nonphysical shapes. By contrast, LBM is able to predict remarkably well the location of air-water interfaces. Nevertheless, given the huge difference in computing time (minutes versus tens of hours) required to run these two models, it is recommended that further research be carried out on the development of both, in parallel.
\end{abstract}

Keywords: Pore-scale, synchrotron X-ray micro CT, soil air-water interfaces, lattice Boltzmann model, morphological model

\section{Introduction}

Over the last decade, a significant body of work has been devoted to the response of soils, and especially of the vast stock of organic matter they contain, to changing climatic boundary conditions or to different agricultural practices. In that context, it has become increasingly clear 
that detailed knowledge is required of the different physical, (bio)chemical, and biological parameters that determine the activity of microorganisms at the microscopic scale. The demand for this information is not new. Several soil microbiologists have pointed it out already for more than half a century [1]. However, not much could be done by way of a response until roughly 15 years ago, when significant technological breakthroughs allowed the geometry of the pore space in soils to be visualized, and the spatial heterogeneity of a number of chemical and mineralogical constituents of soils to be quantified at micrometric scales commensurate with fungal hyphae and even the most minute of bacteria or archaea $[48,40,9,6,7,8]$.

The availability of such microscopic-scale data, combined with the anticipation of further advances in the very near future in terms of both laser and X-ray technologies opening up even brighter prospects, has prompted researchers to develop an array of sophisticated models at the pore scale. Among them, lattice-Boltzmann models (LBM) [29, 20] are able to describe water, solute, and particulate transport in the interstitial space of soils, as well as the shape of air-water interfaces $[13,53]$, without having to invoke the kind of simplifying assumptions about the geometry or topology of soil pores that were typical of earlier generations of models, based on traditional partial differential equations or capillary network idealizations. Similarly, agent- or individual-based models describe quantitatively the growth and metabolism of microorganisms much more realistically than traditional models, based on descriptions of population dynamics, and are able to account in great detail for the effects of the relative spatial distributions of fungi [13], bacteria [27, 30, 18, 15], and the organic matter on which they feed. At the moment, the development of each of these different models is moving forward, in parallel with interdisciplinary efforts to combine them in order to describe various types of micro-scale scenarios and assess the nature of emergent properties of soil systems [13].

As with any type of modeling, part of the work of developing microscopic-scale models of soil processes consists of ensuring that model outputs are adequately approximating the reality they purport to represent. This is particularly important in the case of the spatial distribution of the aqueous phase in the pore space, because this distribution directly impacts the connectivity of liquid-filled pores as well as the rate and extent of air flow, all of which in turn influence directly the distribution and activity of microorganisms. A possible first step to assess the adequacy of a particular model to simulate water distribution in the soil pore space is to compare the results with other models, to make sure that there is general consistency among various alternative descriptions. Vogel et al. [51], for example, compare the outputs of pore network and morphology models with the predictions of a lattice-Boltzmann code, based on structural data relative to a homogeneous sintered borosilicate glass sample. Ideally, however, a further step in the evaluation of a given model should involve the comparison of model outputs to experimental data. Sukop et al. [46] measured the distribution of non-miscible phases in columns of quartz sand at a resolution of $20 \mu \mathrm{m}$, and tried to simulate the results with a multicomponent, multiphase LBM. The outcome is generally satisfying, in terms of bulk concentrations of the different phases, even though detailed analysis of individual pores reveals numerous discrepancies between simulations and observations. A similar exercise has yet to be carried out with an actual soil sample, however.

The fact that attenuation of X-rays by water is quantitatively not very different from that by organic matter makes it very difficult experimentally to distinguish water from soil constituents under many circumstances. To palliate this difficulty, some authors have used other liquids, like decane, instead of water [43], or doped Soltrol, a NAPL, with a marked contrast with water [3], or they have added various contrast agents to the water, to modify its X-ray attenuation $[46,49]$. Another option is to work with porous media that do not contain any organic matter. For example, Brusseau et al. [10] and Culligan et al. [12] show air-water interfaces in 3D computed 
tomography (CT) images of repacked sand and glass beads, respectively, at a resolution of about $12 \mu \mathrm{m}$ using synchrotron X-ray CT. More recently, Andrew et al. [4] show detailed air-water interfaces and are able to successfully measure contact angle of $\mathrm{CO}_{2}$-brine interfaces onto the solid surface of limestone at a small resolution of $2 \mu \mathrm{m}$.

Working with a real soil material, Carminati et al. [11] adopt yet another approach, in that they focus on the water that occupies part of the volume in larger pores, located between aggregates. They are able under these conditions to clearly observe pendular rings of water between two clayloam soil aggregates at a resolution close to $6 \mu \mathrm{m}$. Tippkötter et al. [47] adopt a similar focus, in undisturbed soil samples, and are able with a table-top X-ray CT scanner to visualize the presence of water films coating the inner surfaces of meso- and macropores. These data obtained by Carminati et al. [11] and Tippkötter et al. [47], or equivalent CT data obtained in other soils, could in principle be used to assess whether existing pore-scale models of water distribution in soils are adequate.

In this general context, a first objective of the research reported in the present article was to obtain a new set of 3D images of air-water interfaces at a few $\mu$ m resolution at defined matric potentials in a material constituted of repacked soil aggregates. A second objective was to simulate air-water distribution in this material using two mathematical models to find out which approach is the most promising in order to describe quantitatively the retention of water in soils. The two models, namely a two-relaxation-times (TRT) lattice-Boltzmann model, and the pore morphology model of Monga et al. [35], were chosen because of their contrasting features. The former model is capable of describing very realistically the physics of phase separations, as well as the hydrophobic or hydrophilic properties of the soil solid phase [20] but it presents the relative disadvantage of being very demanding in terms of computational resources. By contrast, the far simpler morphology-based model provides a compact description of pore space and has lower computational costs, but it presents the possible drawback of relying on a simplified description of soil physical laws.

\section{Material and method}

\subsection{Soil preparation}

Soil samples were obtained in the La Cage field site (Versailles, France) from the surface horizon of a silt loamy Albeluvisol [14] with 17\% clay, 56\% silt and $27 \%$ sand. The soil was passed through a $2 \mathrm{~mm}$ sieve, and soil aggregates between 2 and $3.15 \mathrm{~mm}$ in size were collected from the coarse fraction. The gravimetric moisture content of the aggregates was adjusted at $0.205 \mathrm{~kg} . \mathrm{kg}^{-1}$, which was hypothesized to amount to $80 \%$ of 'field capacity' (defined by a matric potential of $-32 \mathrm{kPa}$ or $\mathrm{pF}=2.5$ ). The aggregates were then packed uniformly at a bulk density of $1200 \mathrm{Kg} \cdot \mathrm{m}^{-3}$ into cylinders, $50 \mathrm{~mm}$ in diameter and $40 \mathrm{~mm}$ high. The resulting soil columns were subsequently sawed to about $8 \mathrm{~mm}$ thick slices. Nine $6 \times 6 \times 8 \mathrm{~mm}^{3}$ soil cubes were sampled out of each slice with the help of a razor blade in order to minimize disturbance during cutting. The samples were stored in a cold chamber at $4^{\circ} \mathrm{C}$ before further processing.

\subsection{Miniaturized suction device}

We developed a miniaturized experimental suction device to equilibrate the small soil cubes at fixed matric potentials close to water saturation. The design was specifically conceived to enable measurements at the Synchrotron radiation microtomography (SR- $\mu \mathrm{CT}$ ) facility. Nine syringes, $1 \mathrm{~cm}$ in diameter, were half-filled with $90-125 \mu$ m-diameter glass beads, and subsequently 
connected at the bottom end to a hanging water column. Each soil cube was placed on top of the glass beads in a syringe and after matric potential was equilibrated, the sample was secured in place with a parafilm pellet to prevent any movement during scanning. After that, the syringes were sealed at the top with parafilm. The volume of the air phase above the soil cubes was thus kept as small as possible to prevent evaporation during the tomography scans.

The soil cubes were first equilibrated at a matric potential of $-2 \mathrm{kPa}$ for two days using deaired water. Then, all soil cubes were slowly saturated and were kept equilibrated at $0 \mathrm{kPa}$ for 1 $\mathrm{h}$ in order to remove residual entrapped air. The time duration of this process was initially longer but several cubes collapsed and the whole procedure was repeated with new cubes for a shorter time. Then the syringes, in series of three, were directly equilibrated at specific matric potentials of $-0.5 \mathrm{kPa},-1 \mathrm{kPa}$, and $-2 \mathrm{kPa}$, respectively, for $24 \mathrm{~h}$. For scanning, the syringe was detached from the hanging water column after closing a valve at its bottom tip. Thus, each soil cube was imaged at only one matric potential, with three replicates for each matric potential.

\section{3. $S R-\mu C T$ scans}

Microtomography measurements were carried out at the SR- $\mu \mathrm{CT}$ facility operated by the GKSS Research Centre at HASYLAB (Hamburger Synchrotron Strahlungslabor) belonging to the DESY (Deutsches Elektronen Synchrotron) in Hamburg, Germany. The injected beam is monochromatized by a double crystal monochromator producing a parallel beam with a specific $\mathrm{X}$-ray energy (in this case $30 \mathrm{keV}$ ). Aggregate projections (cubes) were recorded by a CCD camera in $0.2^{\circ}$ steps with rotations from 0 to $180^{\circ}$. The achieved voxel resolution after reconstructing the image projections was $4.6 \mu \mathrm{m}$.

\subsection{Image pre-processing and analysis}

Prior to image analysis and modeling, the reconstructed image datasets were preprocessed using the software package ToolIP with MAVIkit $[16,17]$. First, inscribed cubic subvolumes were extracted to avoid boundary effects at the edges of the sample. The size of the resulting images of about $900 \times 900 \times 500$ voxels was unfortunately too large to carry out LBM computations using the computers at our disposal. Given the fine resolution of the raw data and the fact that our focus was primarily on interaggregate porosity, we opted to decrease the resolution of the images by a factor of two. All image analyses and modeling were thus performed on images with a voxel resolution of $9.2 \mu \mathrm{m}$. To reduce image noise, a rank order median filter was applied. The filter sorts the values of voxels covered by a filter mask (in our case a $3 \times 3 \times 3$ voxel size mask was used) and assigns the median to the considered (central) voxel. To segment the matrix from the detectable pore space a global threshold value was automatically calculated according to Otsu [39] from the gray value histogram by maximizing the intra-class variance of the matrix and pore classes while minimizing the gray value variances within the two classes. The threshold to segment the water- and gas-filled pore regions was selected at the minimum between the two distinct peaks.

The relative fractions of the three phases (gas, water, matrix) were subsequently calculated based on the number of voxels corresponding to each phase. Porosity was calculated as the sum of the relative fractions of gas and water and the water saturation index, $S_{w}$, was equal to the ratio of the relative fraction of water over porosity. Following a procedure suggested by Vogel et al. [52], the pore size distribution (PSD) of the cubes was calculated by a morphological opening of the pore space (i.e, successive erosion and dilation) using 3D spherical structuring elements of increasing radius (from 5 to 45 voxels corresponding to 23 to $207 \mu \mathrm{m}$ ). We used the 
Minimum 3D and Maximum 3D filters of ImageJ software [37] to perform erosion and dilation. Air-water triangulated isosurfaces were generated by the Isocontour tool of ParaView [2] from the extracted 3D data containing the segmented air phase of the images. The surface area was then simply calculated by integration and translated into physical units of $\mathrm{mm}^{2}$ via multiplication with the squared voxel resolution.

\subsection{Two-phase TRT lattice-Boltzmann model}

We used the two-phase single-component two-relaxation-times (TRT) lattice-Boltzmann model because it has intrinsically higher stability, and therefore is better able than singlerelaxation-time schemes to simulate spontaneous phase separation in pore spaces with complex 3D geometries [20]. Such a single-component LBM would not be able to describe two-phase flow displacements with viscous forces representative of an air-water system, but it is nevertheless adequate to describe static conditions of systems at equilibrium, like those in our experiments. Phase separation is simulated by incorporating attractive fluid-fluid short-range interactions to simulate surface tension forces $[44,45]$ and attractive or repulsive fluid-solid interactions $[29,42]$ to simulate hydrophilic or hydrophobic surfaces.

A lattice-Boltzmann model describes a fluid as a collection of fictitious particles that, alternatively, propagate from node to node on a regularly spaced grid, then collide with the particles that end up on the same nodes. The probability of finding a particle at position $\vec{r}$, in one of the microscopic directions envisaged within the lattice, and time $t$ is denoted by $f_{q}(\vec{r}, t)$, where the subscript $q$ is an index associated with the set of microscopic directions. A typical discretization of space is $D 3 Q 19$, in 3 dimensions and with 18 nearest neighbors considered around each node, described by the unit microscopic velocity vectors, $\vec{c}_{q}$. In this case, the subscript $q$ takes on 19 different values (including rest particles).

The mathematical formulation of the model is based on the TRT Boltzmann evolution equation, which describes the relaxation of the probability density function $f_{q}$ to prescribed equilibrium functions $e_{q}^{ \pm}[20,22]$ and is given by:

$$
\begin{aligned}
& f_{q}\left(\vec{r}+\vec{c}_{q}, t+1\right)=f_{q}(\vec{r}, t)+\lambda_{e} n_{q}^{+}+\lambda_{o} n_{q}^{-}+S_{q}^{-}, q=0, \ldots, Q-1 \\
& n_{q}^{ \pm}=f_{q}^{ \pm}-e_{q}^{ \pm}, f_{q}=f_{q}^{+}+f_{q}^{-}, f_{q}^{ \pm}=\frac{1}{2}\left(f_{q} \pm f_{\bar{q}}\right), \\
& \vec{c}_{\bar{q}}=-\vec{c}_{q}, \vec{c}_{0}=\overrightarrow{0}, f_{0}=f_{0}^{+}, f_{0}^{-}=0,
\end{aligned}
$$

The equilibrium functions are given by:

$$
\begin{aligned}
& e_{0}^{+}=e_{0}=\rho-\sum_{q=1}^{Q-1} e_{q}^{+}, e_{q}^{+}=t_{q}^{*} c_{s}^{2} \rho, q=1, \ldots, Q-1, \\
& e_{q}^{-}=t_{q}^{*} \vec{J} \cdot \vec{c}_{q}, q=0, \ldots, Q-1,
\end{aligned}
$$

where $\rho$ and $\vec{J}$ are the macroscopic mass density and momentum flux defined from the local probability density functions $f_{q}$ along:

$$
\rho=\sum_{q=0}^{Q-1} f_{q}, \quad \vec{J}=\sum_{q=0}^{Q-1} f_{q} \vec{c}_{q}
$$


Finally, $\vec{F}_{G}, \vec{F}_{W}$ and $\vec{F}_{g}$ are body forces designed to simulate fluid-fluid, solid-fluid forces and gravity, respectively. They are computed in the source term of the TRT Boltzmann evolution equation, $S_{q}^{-}$.

$$
\begin{aligned}
& S_{q}^{-}=t_{q}^{*}\left(\left(\vec{c}_{q} \cdot \vec{F}_{G}\right)+\left(\vec{c}_{q} \cdot \vec{F}_{W}\right)+\left(\vec{c}_{q} \cdot \vec{F}_{G}\right)\right) \\
& \vec{F}_{G}=-\psi(\vec{r}, t) \sum_{q} t_{G q}^{*} G \psi\left(\vec{r}+\vec{c}_{q}, t\right) \vec{c}_{q}, \psi(\vec{r}, t)=1-\exp (-\rho(\vec{r}, t)) \\
& \vec{F}_{W}=-\psi(\vec{r}, t) \sum_{q} t_{W q}^{*} W s\left(\vec{r}+\vec{c}_{q}, t\right) \vec{c}_{q}, \quad s=\{0,1\} \text { if } \vec{r}=\{\text { fluid, solid }\} \\
& \vec{F}_{g}=-\rho F_{z} \vec{z}, g=F_{z}
\end{aligned}
$$

The parameters $G<0, W$ and $F_{z}$ control the attractive interaction strength, the hydrophobicity of the solid surface and the gravity strength, respectively, whereas $g$ is the gravitational acceleration of the lattice Boltzmann.

Assuming that $c_{s}^{2}$ is an independent parameter (set to $1 / 3$ ), the isotropic weights, $t_{q}^{*}$, for the 3D nineteen-velocities model (D3Q19) used in this study are as follows: $t_{I}^{*}=1 / 6, t_{I I}^{*}=1 / 12$, where the Roman numbers are equal to $\left\|\vec{c}_{q}\right\|^{2}$, so that the $t_{I}^{*}$ apply to orthonormal directions and $t_{I I}^{*}$ to diagonal directions. The weights, $t_{W q}^{*}$ and $t_{G q}^{*}$ are chosen as: $t_{W, 0}^{*}=t_{G, 0}^{*}=0, t_{W, I}^{*}=t_{G, I}^{*}=2$, $t_{W, I I}^{*}=t_{G, I I}^{*}=1[42]$.

Stability conditions of the model impose that the functions $\Lambda_{e}$ and $\Lambda_{o}$ of the two eigenvalues, $\lambda_{e}$ and $\lambda_{o}$, of the TRT collision operator be positive [22]:

$$
\begin{aligned}
& \Lambda_{e}=-\left(\frac{1}{2}+\frac{1}{\lambda_{e}}\right), \Lambda_{o}=-\left(\frac{1}{2}+\frac{1}{\lambda_{o}}\right) \\
& \Lambda_{e o}=\Lambda_{e} \Lambda_{o},-2<\lambda_{e}, \lambda_{o}<0
\end{aligned}
$$

The parameterization of the model was done according to [20]. The eigenvalue, $\lambda_{e}$ was fixed by an arbitrarily value of the kinematic viscosity, $v=1 / 6$ along $v=(1 / 3) \Lambda_{e o}$, for the case of incompressible Stokes flow. The eigenvalue, $\lambda_{o}$ was calculated from a fixed value of $\Lambda_{e o}=3 / 16$.

We used a value of $G=-0.16$ that provides an interface thickness of three nodes of the lattice [20] and selected a value of $W=0.15$ in order to match the fully-wetting fluid conditions (zero contact angle between liquid and solid phases).

The grid of the lattice-Boltzmann model was directly superimposed to the segmented voxels of the 3D SR- $\mu \mathrm{CT}$ images. However, to save computational and memory costs, the calculations were performed only on the nodes of the lattice that were associated with an interstice in the soil.

The liquid-gas distribution was simulated by fixing an initial homogeneous density, $\rho_{i}$ on all the fluid nodes, as spontaneous phase separation is expected to be initiated from interactions with the solid walls. The initial density, $\rho_{i}$, was calculated for each different matric potential from the mass balance equation:

$$
\rho_{i}=\left(\rho_{l}-\rho_{g}\right) S_{w}+\rho_{g}
$$

where $\rho_{g}$ and $\rho_{l}$ are the density of the gas and liquid phases, $\left(\rho_{g}=0.03\right.$ mu.lu ${ }^{-3}$ and $\rho_{l}=2.42$ mu.lu $\left.{ }^{-3}[20]\right)$, respectively, and $S_{w}$ is the water saturation index of each segmented SR- $\mu$ CT images.

Bounce-back conditions were applied at solid boundaries. Since some pores were cut by the edges of our images, we applied hydrophobic conditions $(W=0)$ at the boundary voxels that contained air in the SR- $\mu \mathrm{CT}$ data in order to simulate realistic liquid-gas distributions. Hydrophilic conditions $(W=0.15)$ were applied elsewhere. 
As discussed in [20], we hypothesized that spontaneous phase separation can yield realistic air-water distribution in complex 3D porous medium if the pore space is fully connected. Genty and Pot [20] demonstrated that for the same fixed water saturation, the two-phase LBM can fill two pores of different sizes with water and air differently, depending on whether or not they are connected. If they are not connected, both pores contain air, whereas if they are connected, air is preferentially found in the largest pore as one would expect from capillary theory. The ShanChen LB model thus provides static liquid-gas distribution that can be compared directly with the air-water distribution observed in the 3D SR- $\mu$ CT images.

\subsection{MOSAIC model}

In this approach, we approximate the pore space in the soil by a network of so-called volume primitives $[33,36]$, i.e., simple geometric shapes that can be transformed at will and combined to represent more complex geometries. To do so, we use a geometrical algorithm based on Delaunay triangulation to determine the maximal balls of the pore space segmented from the 3D CT images. Maximal balls are defined as the balls included in the pore space but not included in any other ball included in pore space. Then, we extract a minimal set of the maximal balls in order to obtain a compact representation of the pore space [34].

A mathematical graph is associated with the minimal set of maximal balls. Each node of the graph or ball $B_{i}$ is assigned as many features, $f_{k, i}$, as desired to describe the physical, biological and chemical states of the ball [34], where the subscript $k$ indexes the various features. In this study, we defined one parameter that quantifies the hydration status of each ball. Any given ball $B_{i}$ can be either full of water $\left(f_{1, i}=1\right)$ or empty (full of air, $f_{1, i}=0$ ).

Initially, all nodes are filled by water $\left(f_{1, i}=1\right.$ for all balls $\left.B_{i}\right)$. The final water content distribution is simulated by an iterative drainage procedure, following [50]. In this procedure, the Young-Laplace equation defines the equivalent maximum radius, $r_{\mathrm{eq}}[\mathrm{m}]$, of the pores that are filled with water at a fixed matric potential, $\Psi[\mathrm{kPa}]$ :

$$
\Psi=\frac{2 \sigma \cos (\theta)}{r_{\mathrm{eq}}}
$$

where $\sigma$ is the surface tension of water in contact with air $\left[\mathrm{N} \cdot \mathrm{m}^{-1}\right], \theta$ is the contact angle of water on the solid surface, considered in this study to be zero for a fully wetting fluid.

The original version of the MOSAIC program assumes that balls can empty only if their radius is larger than a threshold value $r_{\text {eq }}$ and if they are connected to the air phase, through wider balls that have already emptied. At each step in the computation, an iterative search is carried out for balls of radius larger than $r_{\mathrm{eq}}$ and in contact with a ball full of air. Balls that meet these requirements are then emptied. The iterative procedure is stopped when equilibrium is reached [34]. In a slightly altered version of the program, this condition on the emptying of balls is lifted. As long as the minimum radius requirement is satisfied, balls are allowed to empty even if they are not connected to a neighboring empty pore.

In fine, like the TRT LBM model, the MOSAIC model also yields spatial static liquid-gas distributions in the soil, which can be directly compared to the air-water distributions observed in the $3 \mathrm{D} \mathrm{SR}-\mu \mathrm{CT}$ images.

\subsection{Pore-scale modeling of air-water interfaces}

Since the main objective of this work was to assess, through comparison with CT data, the ability of two different modeling approaches to simulate the distribution of air and water in soils 
at the pore scale, we chose to focus on single pores to carry out a detailed analysis of model predictions. We selected one cube each out of the series at $-2 \mathrm{kPa}$ and $-1 \mathrm{kPa}$ (cubes bkk03a and bkk04a respectively) on which we performed the simulation of the air-water distribution with the two models. The segmented images were used directly as input data of the pore space for the LBM model whereas a Delaunay triangulation was performed on them by MOSAIC. We observed that the interaggregate porosity consisted of several unconnected pores. The disconnection was due to unresolved microporosity in the SR- $\mu \mathrm{CT}$ tomography datasets. We thus performed the numerical calculations on connected pores by selecting sub-images containing connected porosity only.

To account for the spatial agreement of the position of the menisci between simulations and measurements, the mean absolute error, MAE, was calculated as follows:

$$
\text { MAE }=\frac{1}{n} \sum_{i=1}^{n}\left|s_{i}-m_{i}\right|
$$

where the summation is taken over all pore voxels $n$, and $s_{i}$ and $m_{i}$ are the thresholded densities of the voxels for the simulated and measured images respectively.

\section{Results and discussion}

\subsection{SR- $\mu C T$ scans}

As was also found by Tippkötter et al. [47] and generally unlike in the case of images obtained with table-top, polychromatic X-ray computed tomography scanners [5, 23, 24, 25], the histograms of the 3D SR- $\mu \mathrm{CT}$ images of the soil samples display three distinct peaks (Fig. 1). The height of the peaks in different images cannot be compared to each other, since the sizes of the SR- $\mu \mathrm{CT}$ images differ among samples, but the relative frequencies of each gray level are directly comparable. The attribution of two of the peaks, respectively the leftmost and the rightmost ones, to air and to the mineral components of the matrix (including unresolved pores in the latter case) is straightforward. The middle peak could in principle correspond to either water or organic matter, and caution is therefore warranted to reach a correct interpretation. In some cases, the use of a contrasting agent may be able to tease apart water-filled voxels from those that contain organic matter. However, a preliminary test with one such contrasting agent (KI) indicated that soil structure was significantly modified by it, and that approach was not adopted subsequently. Instead, we assumed as a working hypothesis that all voxels having gray values in the range of the middle peak are indicative of the presence of water, as long as morphological characteristics suggest otherwise.

In all samples, water menisci were clearly visible in the macropores of the soil samples as depicted in Fig. 1, and visual comparison of the original and segmented images, respectively, suggests that the separation of the phases appears reasonable (Fig. 2). Nevertheless, in one sample (bkk09a, data not shown), the segmentation algorithm classified as liquid phase what appears morphologically to be a large piece of organic matter. This pore region was not part of the selected regions of interest used subsequently for the comparison of the modeling results and the SR- $\mu$ CT data.

The porosity values calculated in the SR $\mu \mathrm{CT}$ images are $0.08 \pm 0.02,0.11 \pm 0.02$ and $0.09 \pm 0.02$ at matric potentials of $-0.5 \mathrm{kPa},-1 \mathrm{kPa}$, and $-2 \mathrm{kPa}$, respectively (Table 1). These porosity values correspond to the porosity that can be seen at the resolution of the SR- $\mu \mathrm{CT}$ images, and 

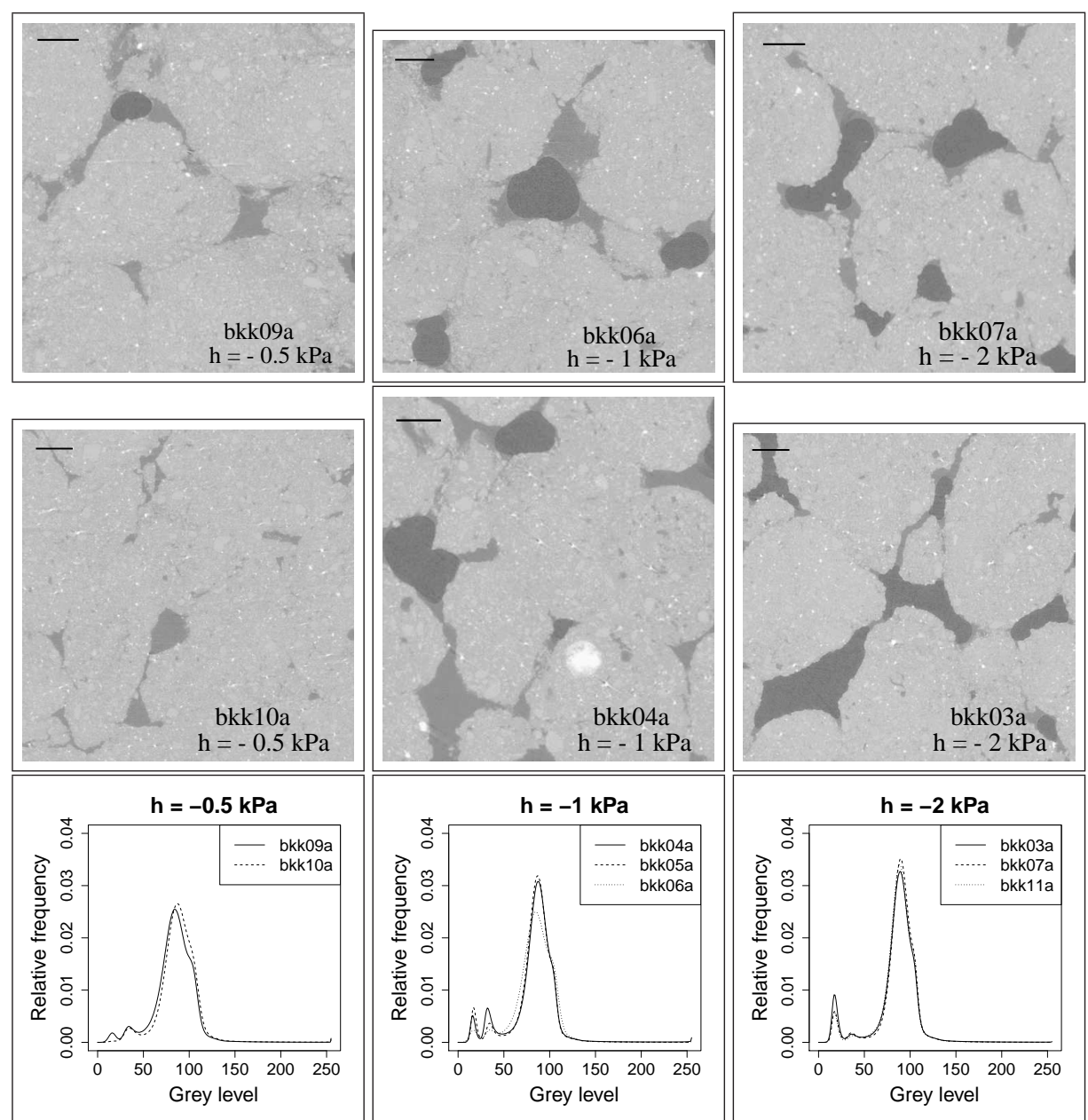

Figure 1: $2 \mathrm{D}$ sections of SR- $\mu \mathrm{CT}$ images of the cubes at the three different matric potentials: $-0.5 \mathrm{kPa}$ (left), $-1 \mathrm{kPa}$ (middle) and $-2 \mathrm{kPa}$ (right) and histograms of the $3 \mathrm{D} \mathrm{SR}-\mu \mathrm{CT}$ images of each cube (bottom figures). In the tomographic sections, black is the air phase, dark gray is the water phase and light gray to white is the matrix phase. The scale bars represent $500 \mu \mathrm{m}$. 


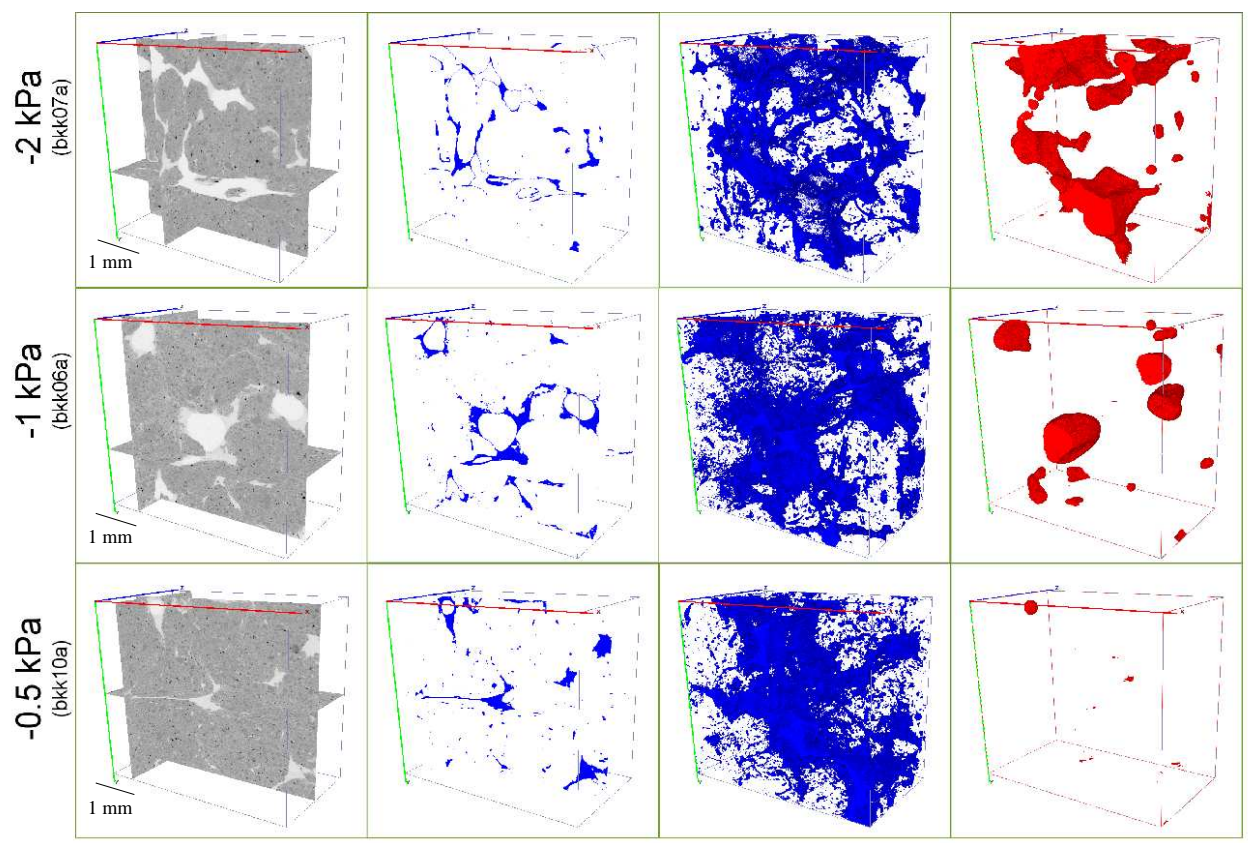

Figure 2: Images of the soil matrix and segmented water and gas phases for selected samples at different matric potentials. From left to right: cross-sectional views of original density maps (shown as gray values with white for the lowest and black for the highest density, respectively); cross-sectional views of water configuration for the same sections; 3D distribution of all water filled pore spaces; 3D distribution of the gas phase. The voxel dimensions of the three reconstructed images, from top to bottom, are $428 \times 451 \times 258,462 \times 458 \times 256$ and $489 \times 474 \times 257$. 
therefore includes only part of the porosity of the soil samples, which hereafter will be referred to as mesoporosity according to Luxmoore's classification [28]. The mesoporosity seems to increase slightly as the matric potential decreases, although there is some scatter in the data since one of the cubes (bkk09a) at $h=-0.5 \mathrm{kPa}$ has a higher porosity. In the latter sample, we observed a piece of organic matter, which was ill-identified as liquid phase by the segmentation algorithm, as mentioned previously. With the particulate organic matter considered as solid phase, the mesoporosity drops by $2 \%$. The trend of mesoporosity being inversely related to the matric potential is confirmed by visual inspection of the original grayscale images, which suggest that the largest macropores are located in the cubes equilibrated at -1 and $-2 \mathrm{kPa}$, compared to the samples equilibrated at $-0.5 \mathrm{kPa}$ (Fig. 3).

These visual observations are confirmed by a pore size distribution analysis, where, following Vogel et al. [52], small- to large-diameter pores are successively removed (Fig. 3). The pore space of cubes equilibrated at a matric potential of $-0.5 \mathrm{kPa}$ consisted predominantly of pores with diameters smaller than $460 \mu \mathrm{m}$, with about $39 \%$ of the pore space corresponding to pores with diameters between 0 and $92 \mu \mathrm{m}$. In contrast, a more homogeneous pore size distribution was observed for the lower matric potentials, with a lower percentage, $(22 \pm 5 \%)$ of pores smaller than $92 \mu \mathrm{m}$ in diameter. Except for one cube (bkk11a, $h=-2 \mathrm{kPa}$ ), where no pores with diameters larger than $460 \mu \mathrm{m}$ were found, the relative volume fraction of larger pores was $16 \pm 5 \%$.

There may be various reasons for these observations. The most obvious one may be that the two samples for the $-0.5 \mathrm{kPa}$ matric potential (bkk09a and bkk010a) collapsed slightly during the initial saturation while this was not the case for the other six samples used for lower matric potentials, which would be a coincidence. On the other hand given the low bulk density $\left(\rho_{b}=\right.$ $1200 \mathrm{~kg} . \mathrm{m}^{-3}$ ) at which aggregates were packed initially, it is also possible that capillary forces between aggregates caused a rearrangement of their geometrical configuration, possibly leading to a local opening of larger pores. This could have led to changes in connectivity of the pore space during desaturation. It is hard to imagine that this mechanism would play a significant role as one would expect instead that capillary forces would pull aggregates together.

Another potential explanation for the observed structural changes may be that aggregates were shrinking to some extent as samples were drained, thus resulting in an increased pore size of the inter-aggregate pore volume. But pore diameters that would drain at -1 and $-2 \mathrm{kPa}$ should be in the order of 300 to $150 \mu \mathrm{m}$ so that probably only a small fraction of intraaggregate pores were drained at all. Regardless of the exact mechanisms that may have resulted in the observed structural changes, these have no bearing on the comparison of models, since both models take the pore space geometry as it was measured, at each individual matric potential, handled separately.

In the SR- $\mu \mathrm{CT}$ images, almost all macropores were full of water at the highest matric potential $(-0.5 \mathrm{kPa})$, and only a small proportion of the pore voxels were filled with air. In one of the samples, some air bubbles were found (bkk09a, Figs. 1 and 2), suggesting that the samples probably contained small amounts of entrapped air, which was not completely removed during saturation. In all samples, macropores progressively filled with air when the matric potential was lowered to $-2 \mathrm{kPa}$, as confirmed by the increase of the relative frequency of air voxels (Fig. 1). At a matric potential of $-1 \mathrm{kPa}$, macropores were approximately equally filled with water and air while at a matric potential of $-2 \mathrm{kPa}$ the air-filled pore space exceeded the water-filled pore space. These observations are in line with the calculations of the average water saturation, $S_{w}$, which decreases from $0.90 \pm 0.13$ for $h=-0.5 \mathrm{kPa}$, to $0.65 \pm 0.11$ for $h=-1 \mathrm{kPa}$, and finally to $0.34 \pm 0.05$ for $h=-2 \mathrm{kPa}$ (Table 1) in agreement with the image histograms (Fig. 1).

The mechanism by which the large pores partially emptied of water as the matric potential was decreased is relevant in the context of the comparison of the two different modeling approaches. 


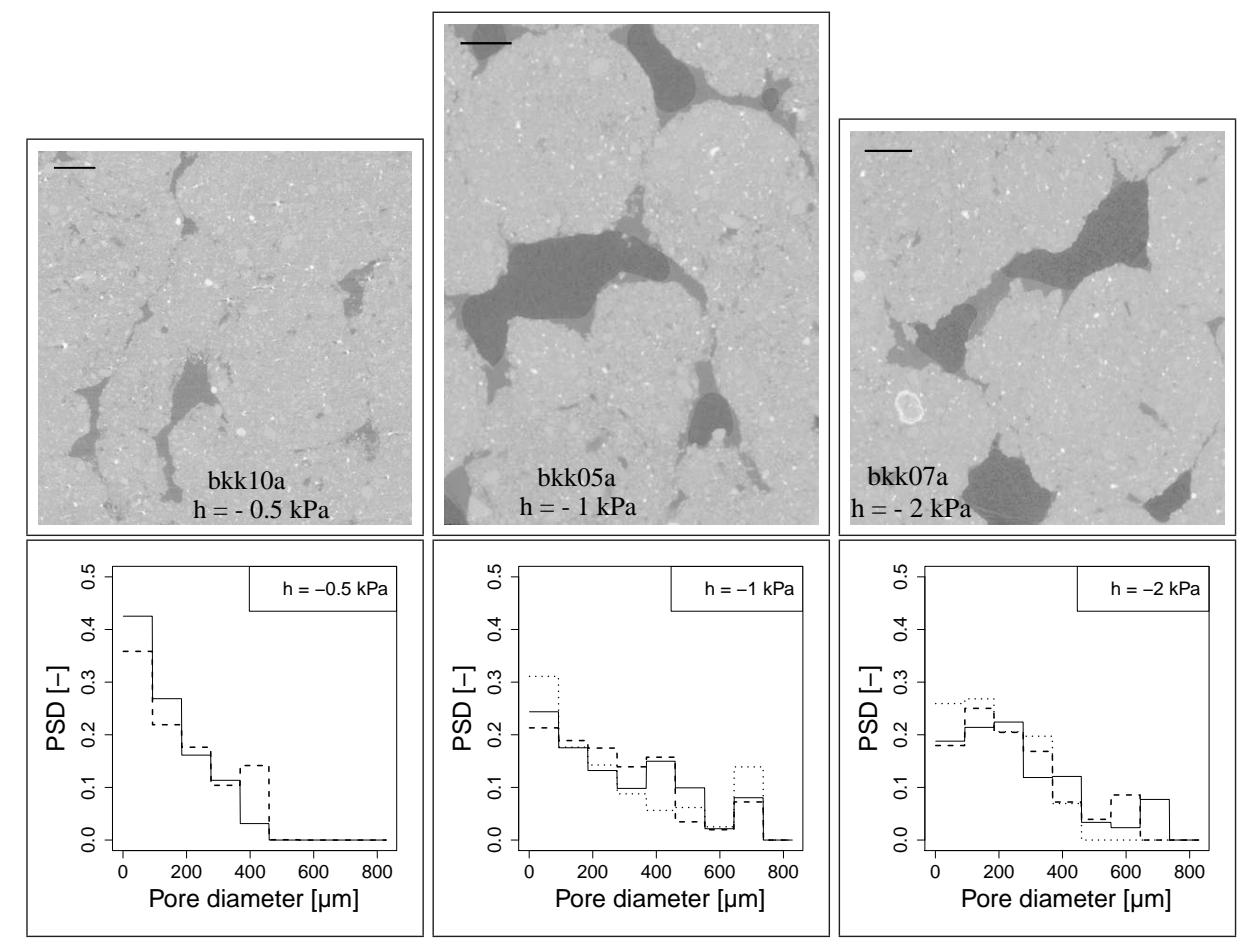

Figure 3: Top: 2D sections of SR- $\mu \mathrm{CT}$ images chosen among three cubes showing the largest interaggregates macropores observed (cubes bkk10a: $-0.5 \mathrm{kPa}$, bkk05a: $-1 \mathrm{kPa}$ and bkk07a: $-2 \mathrm{kPa}$ from left to right, respectively). The scale bars represent $500 \mu \mathrm{m}$. Bottom: PSD curves of all cubes calculated after each morphological opening. Pore diameter is the diameter of the 3D structuring element. The dashed, dotted, and solid lines in the graphs are associated with the different replicate samples.

Table 1: Matric potential, $h$, porosity, $\epsilon$, and water saturation index, $S_{w}$, of the eight SR- $\mu$ CT images.

\begin{tabular}{|c|c|c|c|}
\hline Sample & $h(\mathrm{kPa})$ & $\epsilon(-)$ & $S_{w}(-)$ \\
\hline bkk03a & -2 & 0.10 & 0.28 \\
bkk07a & -2 & 0.08 & 0.38 \\
bkk11a & -2 & 0.07 & 0.35 \\
bkk04a & -1 & 0.13 & 0.72 \\
bkk05a & -1 & 0.10 & 0.53 \\
bkk06a & -1 & 0.09 & 0.71 \\
bkk09a & -0.5 & 0.09 & 0.81 \\
bkk10a & -0.5 & 0.06 & 0.99 \\
\hline
\end{tabular}


Mass flow of the air phase, invading the large pores, would be consistent with the observation of a change in the geometry of the aggregates, and of an opening up of the interstices between them when matric potential was decreased. Although we can not fully explain the responsible mechanism, the PSD curves suggest the creation of macropores with decreasing matric potential (Fig. 3). Aggregates may have moved relative to each other by capillary forces, and thus they may conceivably have enabled air to squeeze in on its way to finding a new stable arrangement. However, if passages large enough existed, they must have closed back down, because when one looks at the full sequence of 2D cross-sections constituting the 3D image, large pores seem surrounded on all sides by small pores, which remained filled with water even at the lowest matric potential, and therefore should not have allowed mass flow of air in the state when the sample was in equilibrium with the applied matric potential. Again, the PSD curves depicted a reduction by half of the pore volume occupied by pores of an equivalent diameter inferior to $92 \mu \mathrm{m}$ when matric potential was decreased supporting thus the idea of a closure of such passages. In loose homogeneous loess soils, Peth et al. [41] also observed similar modification of soil structure with an increase of large pores and a concomitant closure of small pores during drying. In loose packing where soil is easily compressible, the opening of new macropores could more easily induce a closure of smaller pores as a response to hydraulic stress.

Perhaps a more plausible explanation for the presence of pockets of air in the large pores is that nucleation occurred and bubbles formed that increased in size until they eventually filled the pores. Little has been written about nucleation in soils [32, 38, 19]. It is well-known that this process is very hard to eliminate in tensiometers tubes, where the slightest impurity, a piece of dust or a spot of grease on the inside surfaces of the tubes, is enough to cause nucleation and lead the tensiometers to fail [31]. In soils, one would expect nucleation points to be extremely numerous, from clay particles or particulate organic matter to bacterial cells or fungal hyphae, located in the lumen of pores or on solid surfaces. For nucleation to occur, air needs to be dissolved in the liquid phase. Even though, in our experiments, the water with which the soil samples were filled was initially de-aired, it is likely that some air managed to dissolve again in the liquid phase by the time the samples were scanned, a few days later.

Since nucleation does not seem to have limited the appearance of bubbles or air pockets in the soil samples, all that is needed to account for their presence in the pores as shown in Figs. 1 and 3 is that the size of pores be sufficient to accommodate air pockets that are stable at the matric potentials applied. Miller [32] considers that air bubbles with a diameter smaller than the critical diameter predicted by the Young-Laplace equation at a given matric potential would be unstable, and eventually collapse. Therefore, based on classical capillary theory as a rule of thumb, pores need to be of about the size of this critical diameter, i.e., 280 and $150 \mu \mathrm{m}$, respectively, at matric potentials of $-1 \mathrm{kPa}$ and $-2 \mathrm{kPa}$, for air bubbles to persist. These dimensions are consistent with the sizes of most pores in which bubbles are located (Figs. 1 and 3).

\subsection{Pore-scale modeling of air-water interfaces}

For the cube bkk04a, in the soil sample equilibrated at $-1 \mathrm{kPa}$, ROI p1bkk04a of size $169 \times 223$ $\times 210$ voxels has a volume of $6.2 \mathrm{~mm}^{3}$ and contains a pore of approximately ovoidal shape in its inner part with elongated extensions in several directions. For the cube bkk03a, equilibrated at $-2 \mathrm{kPa}$, two regions of interest (ROI), p1bkk03a and p4bkk03a, were selected. Their sizes are, respectively, $132 \times 157 \times 90$ voxels and $112 \times 208 \times 90$ voxels, corresponding to volumes of 1.4 $\mathrm{mm}^{3}$ and $1.6 \mathrm{~mm}^{3}$. ROI p1bkk03a involves a single, roughly rhombohedrical pore, whereas ROI p4bkk03a comprises two elongated pores connected by a narrow constriction (throat). The two pores p1bkk04a and p1bkk03a share a relatively simple morphology (close to a rounded shape). 
By contrast, pore p4bkk03a has a more complex geometry, with throats connecting larger pores. These contrasting morphologies allowed us to test the models under different conditions.

\subsubsection{Modelling at $-1 \mathrm{kPa}$}

The water saturation index of the pore space in ROI p1bkk04a is estimated from the thresholded 3D image to be around 0.45. At the matric potential of $-1 \mathrm{kPa}$, the central part of the main pore, with a maximal diameter evaluated by MOSAIC to be $700 \mu \mathrm{m}$, is occupied by air (Figs. 4 and 5), in spite of the fact that the narrow, water-filled extensions of the central pore should have prevented any mass movement of air.

To simulate the air-water distribution with the LBM model, an initial average density was adopted, corresponding to the saturation level measured on the SR images, according to a procedure described by Genty and Pot [20,21]. Then the model initiated a phase separation and the position of water menisci was determined by equilibrium between cohesive forces in the liquid phase, as well as wetting- and gravity forces.

Simulation results suggest that for this ROI p1bkk04a, the distribution of water and air predicted by the LB method agrees remarkably well with experimental observations. Discrepancies are very minor, like an air pocket predicted in a small pore in section $z=22$ (left column in Fig. 4), which is not in the SR image.

A 3D perspective view of the pore, as in Fig. 5, allows a better visual inspection of the correspondence between SR- $\mu \mathrm{CT}$ observations and LBM predictions. In smaller cylindrical-shaped pores, LBM predicts the presence of two separate small air pockets that are not seen in the SR$\mu \mathrm{CT}$ images, whereas in another small pore, close to plane $(x=169)$, LBM does not predict the presence of air, which SR- $\mu \mathrm{CT}$ detected. Nevertheless, these discrepancies are limited in scope, and overall the shape and location of the air-water interface in this ROI are close to the reality as revealed by SR $-\mu \mathrm{CT}$ images. This goodness-of-fit translates into a very low mean absolute error, equal to 0.08 .

In the case of MOSAIC, the Young-Laplace equation determines if the geometric primitives (balls) representing the pore space can in principle empty at the matric potential that is applied on the soil sample. Whether or not a pore that could in principle empty indeed does so depend in practice on the connectivity of the air phase [26]. In this particular instance, no drainage of the large pore could take place because it is not accessible to air, i.e., the throats full of water surrounding this pore cannot empty since the matric potential applied is not low enough for that. The 3D perspective views of the scanned image (Fig. 5) show clearly that, in ROI p1bkk04a, the central pore containing air is enclosed by throats full of water in all spatial directions. As a matter of fact, since a crucial condition for bulk air penetration is not met, MOSAIC fails to predict the presence of air in this case (data not shown).

When the constraint relative to the connectivity of the air-filled pore space is lifted, i.e., when the balls envisaged by MOSAIC are allowed to empty independently of each other, MOSAIC predicts filling of the large pore in ROI p1bkk04a with air (third row in Fig. 4) with an equal amount of air $\left(S_{w}=0.45\right)$. The air-water interfaces agree well with the observed ones in the SR$\mu \mathrm{CT}$ images, although the MAE is higher, at 0.16 , than that obtained with LBM. In the small pore in section $z=22$, MOSAIC does not predict the presence of air, in contrast to LBM. However, the air-water interface has an unphysical, globulous appearance, compared to the smooth shape predicted by LBM and observed in the SR- $\mu \mathrm{CT}$ data. In one instance, at the bottom of the air pocket in section $z=64$, MOSAIC emptied one ball resulting in a manifestly unphysicallooking shape for the predicted air-water interface. The latter features are undoubtedly due to the discretization of the pore space as a collection of geometric primitives (balls), as nicely evinced 


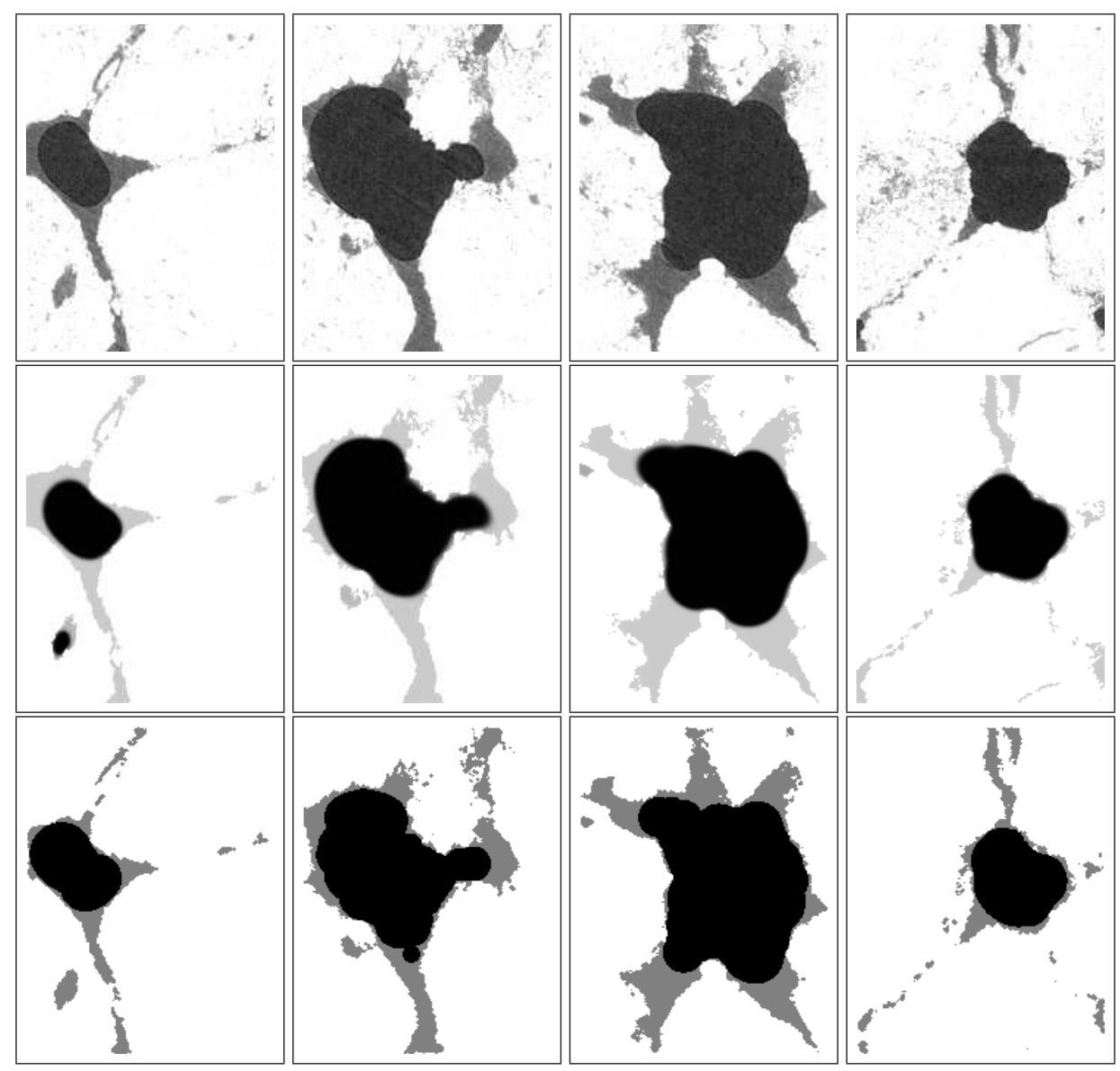

Figure 4: Comparison between SR- $\mu$ CT (first row), LBM (second row) and MOSAIC (fourth rows) of the air-water distribution in the connected pore space of ROI plbkk04a at different vertical sections $\mathrm{z}=22,64,106$ and 148 (from left to right). The water saturation index of the pore is 0.45 . Solid phase is in white, water is in light gray and air is in black.

by the surface rendering of the air-water interfaces in Figure 6. By contrast, LBM calculates the balance between the different forces acting on water particles and simulates minimum interfacial areas. The air-water interfacial area that MOSAIC predicts for the pore depicted in Figure 6 is $6.4 \mathrm{~mm}^{2}$, slightly higher than the value of $5.9 \mathrm{~mm}^{2}$ found with LBM, confirming to some extent the unphysical shape of the interfaces predicted by MOSAIC. The interfacial area calculated in the segmented SR- $\mu$ CT image is intermediate, with a value of $6.1 \mathrm{~mm}^{2}$.

\subsubsection{Modelling at $-2 \mathrm{kPa}$}

Visual inspection of the pore space in Figs. 7-10 suggests that the inner diameter of the central pore of p1bkk03a is larger than that of the pores in p4bkk03a. Indeed, MOSAIC provides an estimate of the largest pore diameter in each ROI: $382 \mu \mathrm{m}$ for p1bkk03a, and $204 \mu \mathrm{m}$ for p4bkk03a. As expected, this translates into very different degrees of water saturation, of 0.20 and 0.49 , respectively, at the matric potential of $-2 \mathrm{kPa}$. 


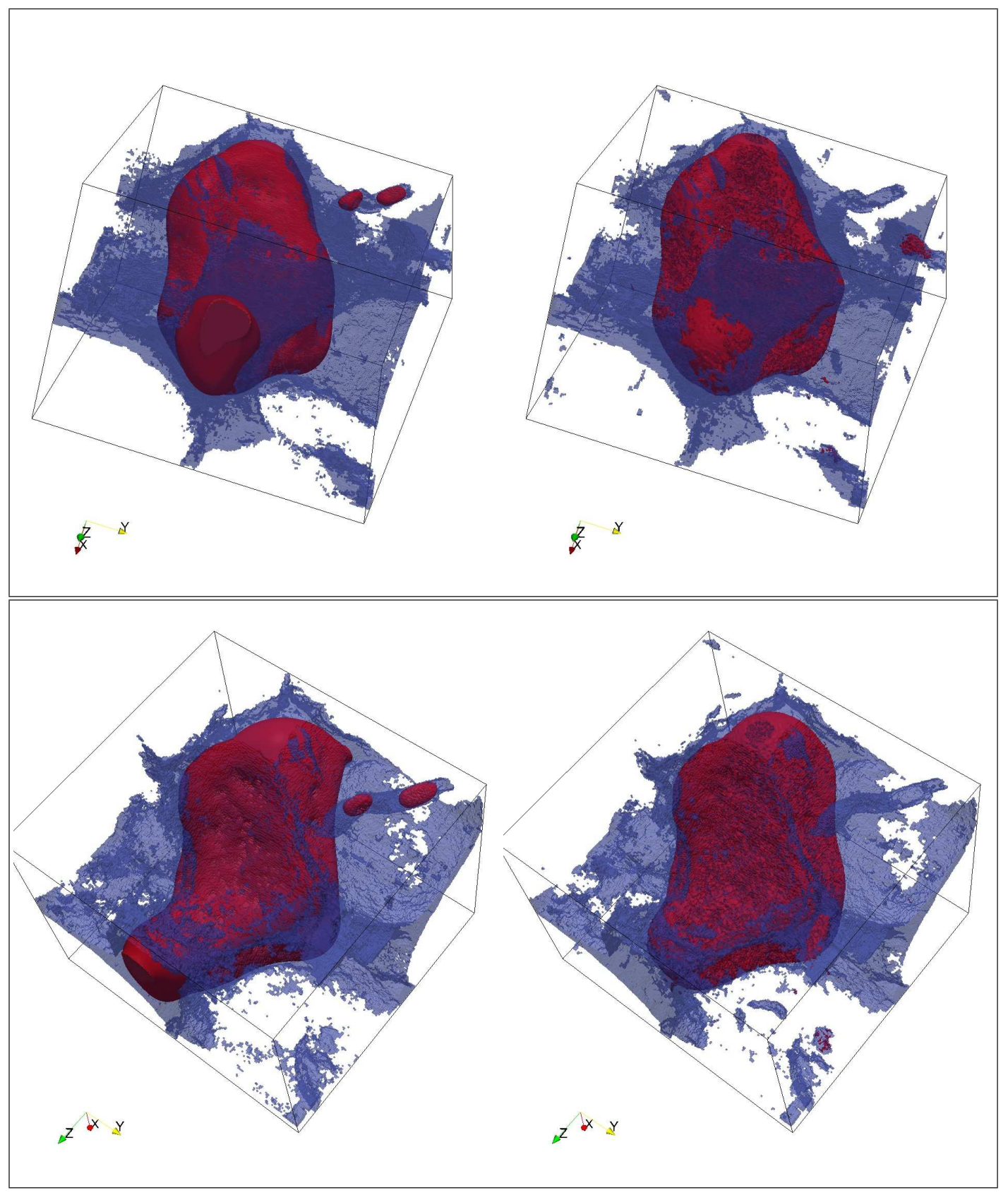

Figure 5: Region of Interest p1bkk04a viewed from two different perspectives, respectively along the z-axis (top) and the $\mathrm{x}$ axis (bottom). LBM predictions are on the left, and synchrotron X-ray CT images on the right. The solid phase is not represented, whereas water is in blue and air is in red. (see article online for color version of this figure) 


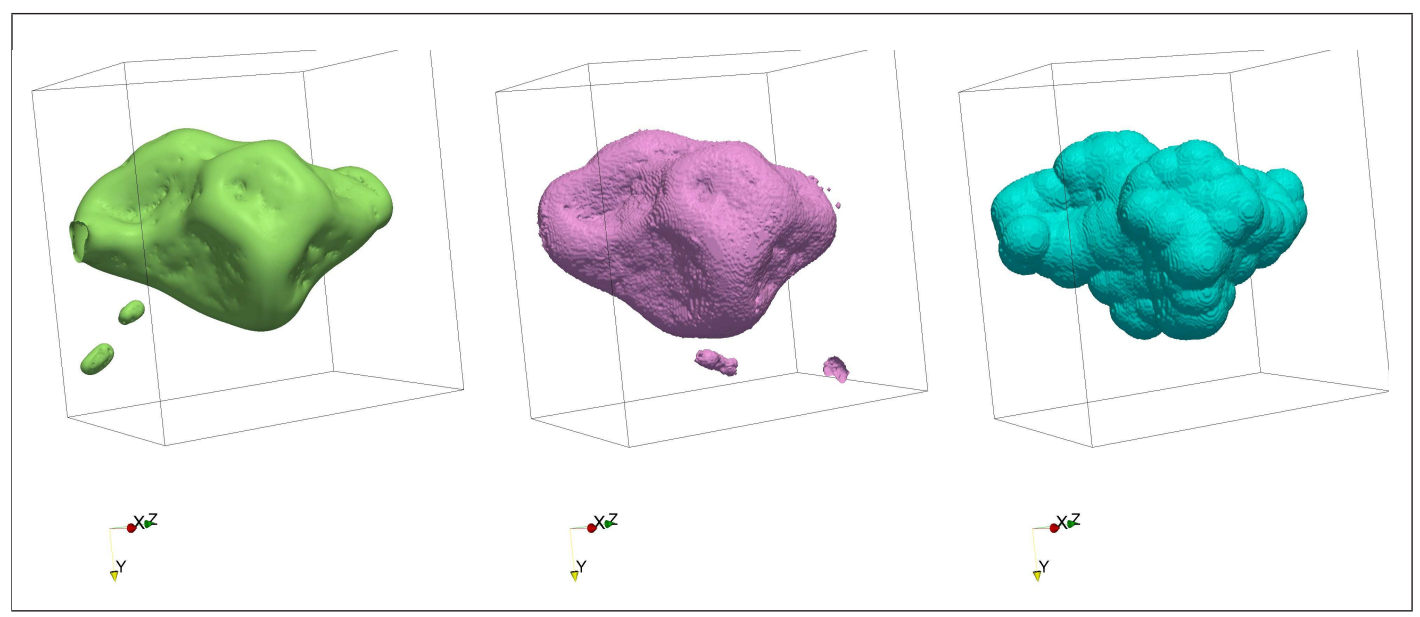

Figure 6: Air-water interfacial surfaces of Region of Interest p1bkk04a for LBM (left), synchrotron X-ray CT images (middle) and MOSAIC (right). (see article online for color version of this figure)

Simulation results suggest that for the more regular shape of the pore of p1bkk03a, the distribution of air and water, as well as the location of the air-water interfaces, predicted by the LB method agrees remarkably well with the measured one (Fig. 7 and 9). For p4bkk03a ROI, again a generally good agreement is observed between LBM predictions and SR- $\mu$ CT measurement, although more water is found in the top center part of the pore (see sections 30 and 39 of Fig. 8) and an air pocket is predicted to be present in the bottom left part of the pore (see section 39 of Fig. 8), when in fact none is found in the corresponding SR- $\mu \mathrm{CT}$ image. These minor discrepancies are perhaps clearer in the 3D graphs (Fig. 10), where one can see the simulated air pocket connected to the boundary ( $z=0$ plane) of the cube (top figures of Fig. 10), and where the air pocket close to plane $(y=0)$ is larger than in the measured image.

For p1bkk03a, an MAE of 0.05 confirms the excellent agreement between the LBM-predicted and SR- $\mu$ CT images (Fig. 7), whereas a larger value of 0.13 for p4bkk03a (Fig. 8) indicates a higher degree of mismatch.

By contrast, the MOSAIC model did not manage to reach the measured levels of saturation: water saturations of 0.32 and 0.70 were obtained for p1bkk03a and p4bkk03a (third row in Figs. 7 and 8). The calculated MAE values are 0.23 and 0.37 , respectively.

In order for the location of the air-water interfaces predicted by MOSAIC to be closer to SR- $\mu$ CT observations, a different approach needs to be adopted, namely simply emptying balls starting from the largest radii down to the lower ones until a satisfactory water saturation level is obtained. For ROI p1bkk03a, this point is reached when the threshold diameter of the balls is equal to $73.6 \mu \mathrm{m}$, at which point the level of water saturation in the ROI is 0.19 (fourth row in Fig. 7). For ROI p4bkk03a, a water saturation of 0.50 is reached when the threshold diameter of the balls is equal to $112 \mu \mathrm{m}$ (fourth row in Fig. 8). The predictions made with the MOSAIC model are significantly better under these conditions. In p4bkk04a, in some places, MOSAIC simulates the distribution of air and water better than LBM, with an amount of water in the top center part of the pore that compares more favorably with that in the SR- $\mu \mathrm{CT}$ images (sections 30 and 39 of Fig. 8). In addition, unlike LBM, MOSAIC does predict the presence of an air bubble in the bottom left part of the pore (section 39). In other places, MOSAIC is in disagreement 


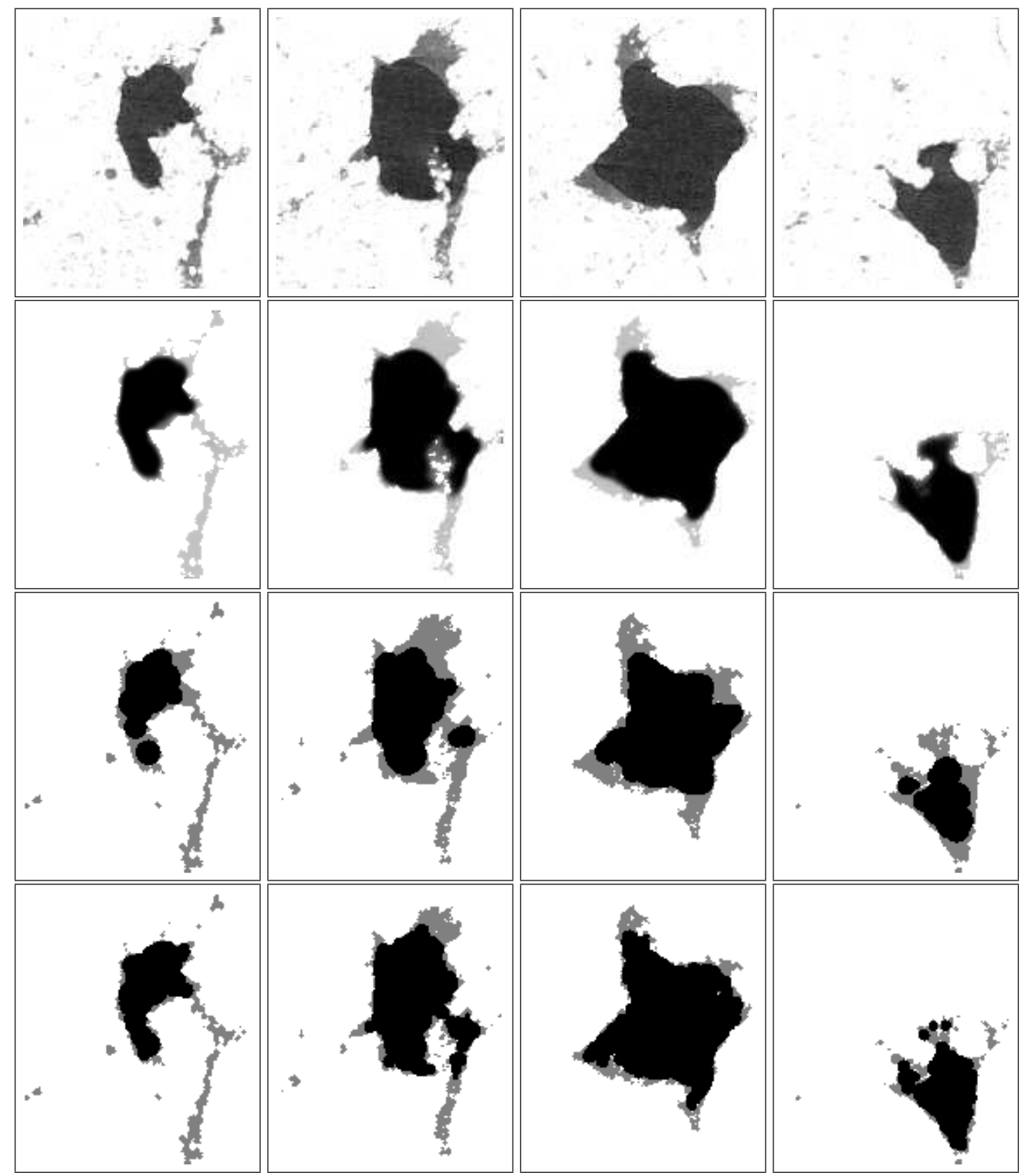

Figure 7: Comparison between SR- $\mu$ CT (first row), LBM (second row) and MOSAIC (third and fourth rows) of the air-water distribution in the connected pore space of ROI p1bkk03a at different vertical sections $z=18,24,34$ and 65 (from left to right). The water saturation index of the pore is 0.20 . Solid phase is in white, water is in light gray and air is in black. The third row corresponds to a threshold diameter value of $150 \mu \mathrm{m}$ corresponding to $h=-2 \mathrm{kPa}$ (resulting in $S_{w}=0.32$ ) while the fourth row corresponds to a lower threshold value of $73.6 \mu \mathrm{m}$ (resulting in $S_{w}=0.19$ ). 


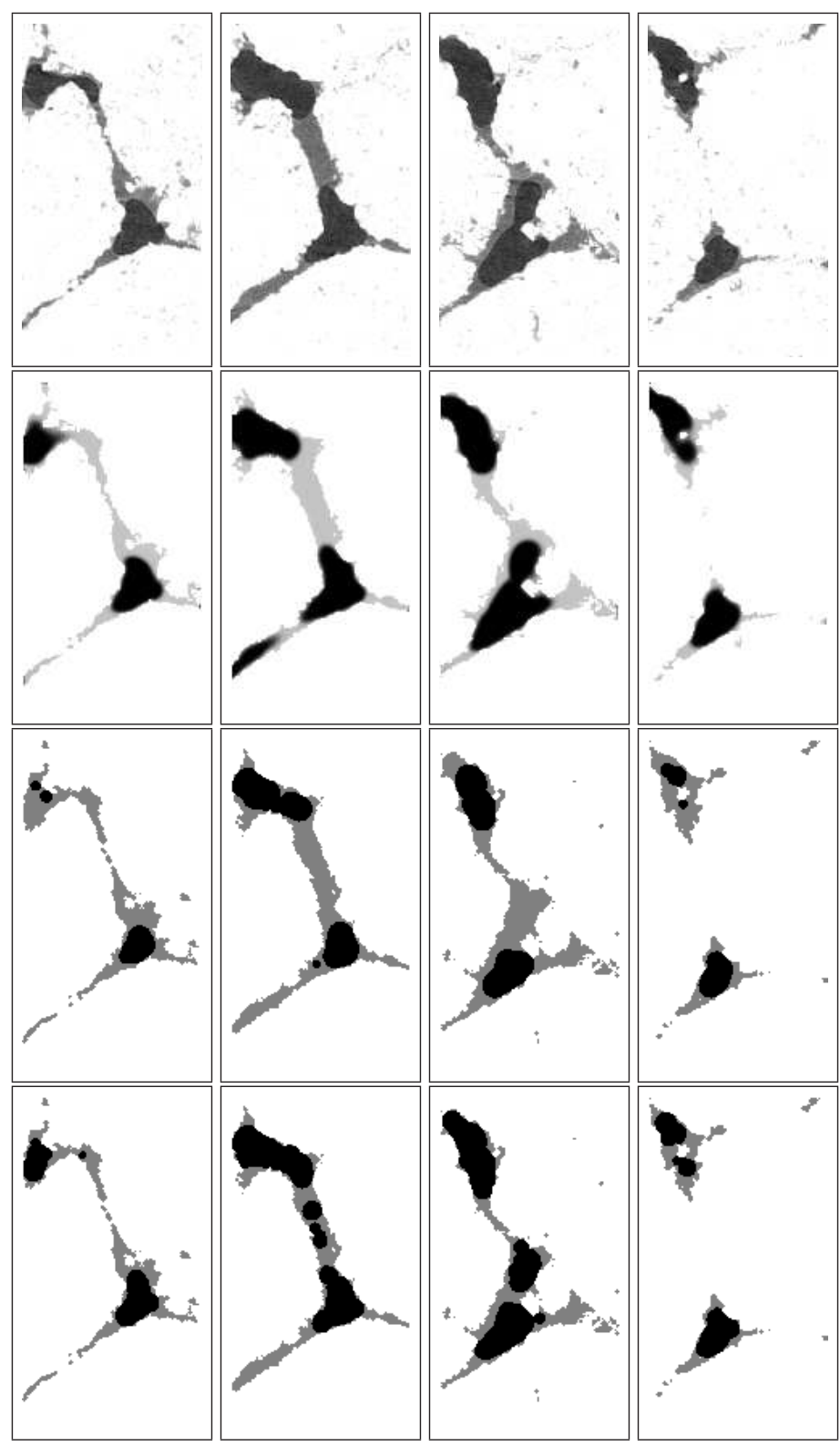

Figure 8: Comparison between SR- $\mu$ CT (first row), LBM (second row) and MOSAIC (third and fourth rows) of the air-water distribution in the connected pore space of ROI p4bkk03a at different vertical sections $z=30,39,54$ and 63 (from left to right). The water saturation index of the pores is 0.49 . Solid phase is in white, water is in light gray and air is in black. The third row corresponds to a threshold diameter value of $150 \mu \mathrm{m}$ corresponding to $h=-2 \mathrm{kPa}$ (resulting in $S_{w}=0.70$ ) while the fourth row corresponds to a lower threshold value of $112 \mu \mathrm{m}$ (resulting in $S_{w}=0.50$ ). 


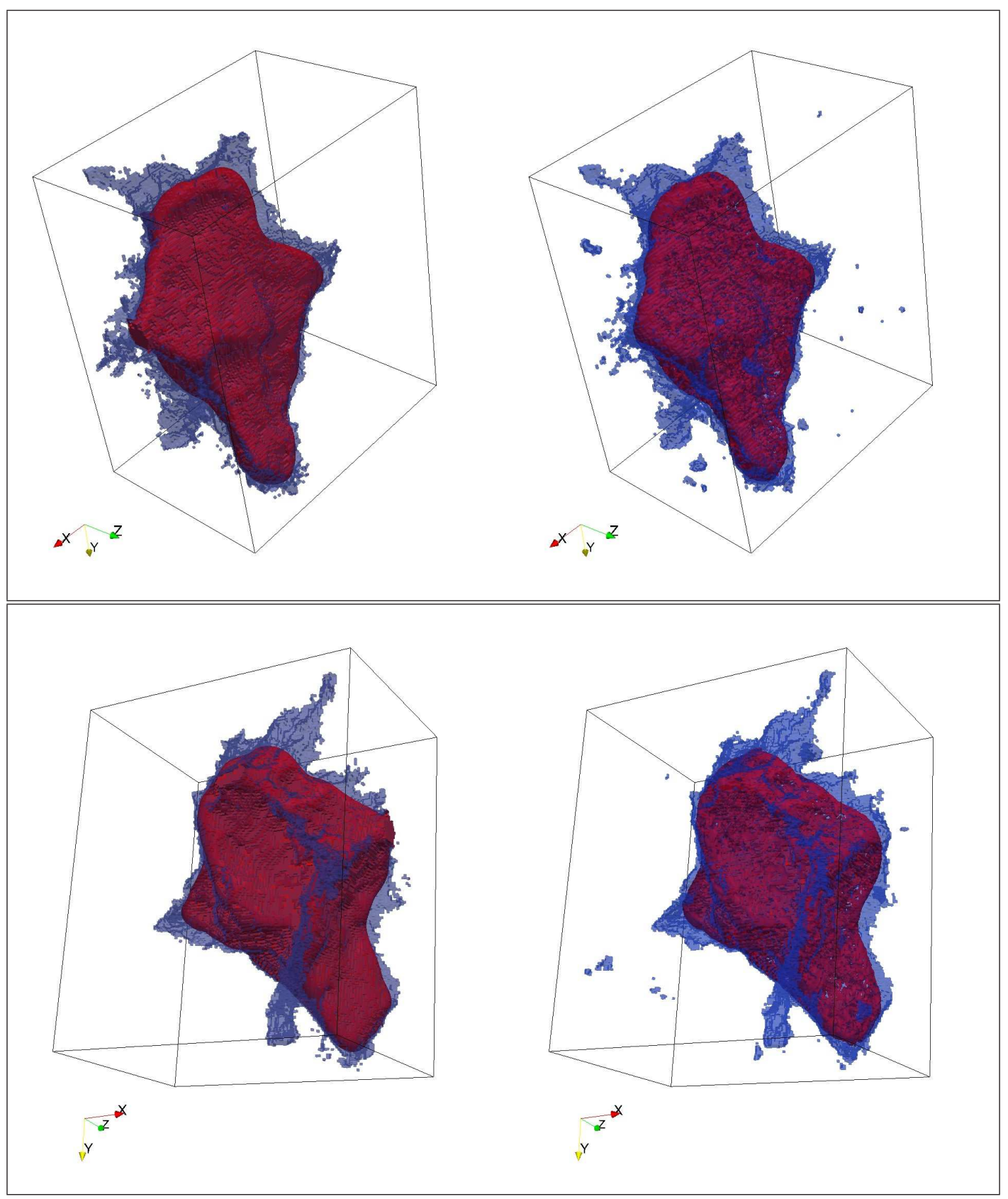

Figure 9: Region of Interest p1bkk03a viewed from two different perspectives, respectively diagonally in the $\mathrm{x}-\mathrm{y}$ plane (top) and along the $\mathrm{z}$ axis (bottom). LBM predictions are on the left, and synchrotron X-ray CT images on the right. The solid phase is not represented, whereas water is in blue and air is in red.(see article online for color version of this figure) 


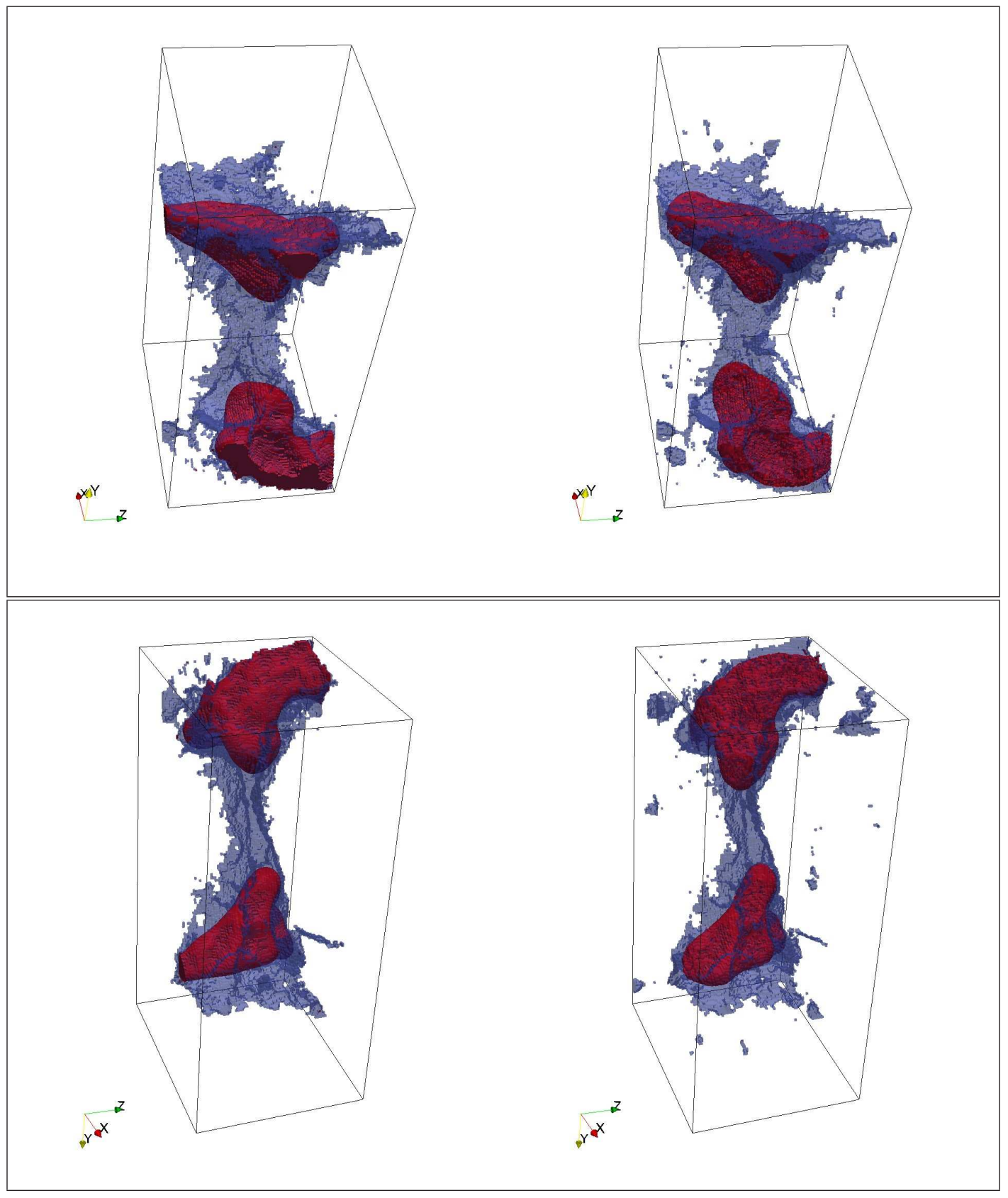

Figure 10: Region of Interest p4bkk03a viewed from two different perspectives, respectively looking downward along the $\mathrm{y}$ axis (top) and upward along the same axis (bottom). LBM predictions are on the left, and synchrotron X-ray CT images on the right. The solid phase is not represented, whereas water is in blue and air is in red (see article online for color version of this figure). 
with SR- $\mu \mathrm{CT}$ images, for example when it predicts the presence of air pockets in the middle of the central part of the pore (section 39), and a discontinuity of the liquid phase in the bottom part of the pore (section 54). Finally, by contrast to LBM, an unphysical shape of the air-water interface is clearly noticeable due to the ball discretization. The calculated MAE is 0.18 and 0.30 for p1bkk03a and p4bkk03a, respectively, higher than the error calculated for the LBM results. The air-water interfacial areas are 2.5 and $1.8 \mathrm{~mm}^{2}$ for p1bkk03a and p4bkk03a, respectively, slightly higher than the corresponding values of 2.2 and $1.7 \mathrm{~mm}^{2}$ obtained with LBM, which in turn are very near the values of 2.1 and $1.7 \mathrm{~mm}^{2}$, respectively, found for the measured SR- $\mu \mathrm{CT}$ data.

\subsubsection{Discussion of modeling results}

In order for the MOSAIC model to predict the location of air-water interfaces even approximately, the restriction imposed by capillary theory that only pores connected physically to airfilled pores can drain has to be lifted. This observation seems to lend credence to the idea that the presence of gas pockets in the SR- $\mu \mathrm{CT}$ images is due to some kind of nucleation, followed by the growth of air bubbles out of gases dissolved in the aqueous phase. Actually, the remarkable fit of LBM predictions with observations to some extent and very indirectly, also supports the same scenario. Clearly, since the LBM scheme that was adopted in the research involves only one component, there is no way it could describe explicitly the processes causing the coalescence of dissolved gases into bubbles. Indeed, only (virtual) particles associated with water are considered explicitly by the LBM scheme. When calculations lead these particles to separate into a dense phase and a light phase in specific portions of the pore space, this phase separation is the manifestation only of a balance of the different forces acting on water particles, in particular forces of adsorption of these particles on pore walls. However, for all practical purposes, the equilibrium state associated with this phase separation is phenomenologically indistinguishable from a situation in which a bubble would have formed in a pore, and the volume of this bubble would no longer change through degassing of the liquid phase.

If one considers that this scenario of the spontaneous growth of air pockets in the pore space is valid, then the fact that MOSAIC is able to predict the presence of air, and in one instance the same amount of air, suggests that the representation of the pore space via simple geometric primitives is an acceptable approximation. The computation carried out by the LBM and MOSAIC both require knowledge of the degree of saturation of the pores, a condition that poses practical problems. Indeed, it is clear from comparison of the results for the two ROIs at a matric potential of $-2 \mathrm{kPa}$ that even within a single soil sample, different pores have different degrees of saturation. However, Genty and Pot [20] have shown that, as long as pores are connected in a soil sample, the only piece of information about the degree of saturation that LBM needs as input relates to its value for the network of pores as a whole, not that of each individual pore separately, as with MOSAIC. This is a definite advantage of LBM over MOSAIC, in that knowledge of the actual degree of saturation of a soil is generally available in practice whenever one might want to simulate the distribution of water in the pore space, at least under static conditions.

Nevertheless, it may be possible to rethink somehow the discretization of the pore space in MOSAIC to make the model better able to simulate the distribution of water and air in soils. One major incentive to continue working along that path is that, compared to the more accurate LBM model, MOSAIC is blazingly fast. On a similar type of computer (typical desktop computer with two $2.6 \mathrm{GHz}$ processors), running the LBM program on one of the ROIs considered earlier takes between 2 and $19 \mathrm{~h}$, while exactly the same cases take about 5 min when run with MOSAIC. The considerable time savings that ensue may be extremely useful when one tries to work with larger 
soil samples, as is very likely to be increasingly the case in years to come, in order to resolve the question of how to upscale microscopic simulations to larger scales (e.g., [8]).

\section{Conclusion}

A first result of the research described in this article is a new experimental data set of 3D images of air-water interfaces obtained in a soil via synchrotron X-ray computed tomography at different matric potentials $(-0.5,-1$ and $-2 \mathrm{kPa})$. The high quality of the images allows a clear visualization of the water menisci without using a dopant for water. These images clearly show that soil pores can desaturate even in situations where capillary theory predicts that they should not. Indeed, pores partially filled with air in some of the images are surrounded by smaller pores that remain consistently water-saturated, in contradiction with capillary theory. A likely explanation for this observation is the process of nucleation and the subsequent growth of bubbles as dissolved gases are released from solution. These observations of non-capillary desaturation of pores have significant practical consequences in that any prediction of water saturation of a soil using capillary theory, without any allowance for nucleation and bubble growth, is likely to overestimate the amount of water that is actually present in the system.

A second result is illustrated in Figs. 4 - 9. For the first time, predictions made with two computer models of the distribution of water in soil samples equilibrated at different matric potentials are compared with experimental data, obtained via synchrotron X-ray computed tomography. Results of this comparison indicate that the two models do not fare equally. MOSAIC, a model based on the representation of pore space through a collection of geometrical primitives and application of capillary theory, fails to account for experimental observations as long as pores are restricted to drain only when some contiguous interstice is already full of air. This is a significant observation, in the sense that this same restriction underlies most capillary network models used routinely to describe the retention or movement of water in variably-saturated porous media. When this assumption is lifted, i.e., pores are allowed to drain regardless of the status of their neighbors, the performance of MOSAIC improves noticeably. On a case by case basis, it is possible to obtain a reasonable agreement between measured and predicted locations of air-water interfaces with MOSAIC if the critical diameter at which pores empty is lowered to reproduce the experimentally measured degree of water saturation. However, this procedure is clearly arbitrary, and renders the model unsuitable for practical use in its current version. In addition, predicted air-water interfaces are oddly shaped, likely as a consequence of the discretization of the pore space with geometrical primitives, which then determine the configuration of the interfaces between air and aqueous phases. Even though this does not translate into very different air-water interfacial area values, compared with LBM predictions, processes dependent on the geometry of air-water interfaces would be affected.

From a modeling standpoint, the most substantial result of the research described in this article undoubtedly relates to the remarkably accurate prediction of air-water interfaces by the latticeBoltzmann model. In very few cases, LBM predicts the presence of air in pores where none is observed experimentally, whereas in other cases, the opposite occurs. Nevertheless, these discrepancies are very minor. Overall, the results of the comparison are remarkably good, with a high level of agreement between measured and predicted locations of air-water interfaces. These observations suggest that LBM-based models can be used reliably to determine where water is located in the pore space of soils, information that is crucial to describe properly the activity of soil bacteria, archae, and fungi. One drawback of the LBM is the very long time that computations take, of the order of $20 \mathrm{~h}$ for the longest simulations carried out in this research. 
With techniques like domain decomposition, the use of multiple processors, and computations using graphic processors (GPUs) instead of CPUs, it will probably be possible to speed the computations up drastically in years to come, but it is likely that LBM will always take far longer to run than blazingly-fast MOSAIC, whose computing times are of the order of a few minutes on a desktop computer. Therefore, for the time being, it seems wise to pursue further development and testing of both LBM and MOSAIC approaches in parallel.

\section{Acknowledgments}

This work was supported in part by a bilateral French-German PROCOPE Project and the French ANR project ANR-09-SYSCOMM MEPSOM. The authors also thank HASYLAB (Hamburger Synchrotron Strahlungslabor) at DESY (Deutsche Elektronen-Synchrotron) for access and the Helmholtz-Association for supporting the use of the radiation source under contract no. I-2011-0228. M. Ogurreck gratefully acknowledges financial support from the German Research Foundation (DFG) via SFB $986 \mathrm{M}^{3}$, project Z2.

\section{References}

[1] Alexander M. Biochemical ecology of soil microorganisms. Annu Rev Microbiol, 1964;18:217-250.

[2] Ahrens J., Geveci B., Law C. ParaView: An End-User Tool for Large Data Visualization. Visualization Handbook, Elsevier, 2005, ISBN-13:978-0123875822.

[3] Al-Raoush R. Experimental investigation of the influence of grain geometry on residual NAPL using synchrotron microtomography. J Contaminant Hydrol, 2014;159:1-10.

[4] Andrew M., Bijeljic B., Blunt M.J. Pore-scale contact angle measurements at reservoir conditions using X-ray microtomography. Adv Water Resour, 2014;68:24-31.

[5] Baveye, P.C., Laba, M., Otten, W., Bouckaert, L., Dello Sterpaio, P., Goswami, R.R., Grinev, D., Hu, Y., Liu, J., Mooney, S., Pajor, R., Sleutel, S., Tarquis, A., Wang, W., Wei, Q., Sezgin, M., 2010. Observer-dependent variability of the thresholding step in the quantitative analysis of soil images and X-ray microtomography data. Geoderma, 2010;157:51-63.

[6] Baveye, P.C., Rangel, D., Jacobson, A.R., Laba,M., Darnault, C., Otten, W., Radulovich, R. From dust bowl to dust bowl: Soils are still very much a frontier of science. Soil Sci.Soc.Am.J. 2011;75:20372048.

[7] Baveye, P.C., Laba, M. Moving away from the geostatistical lamppost: Why, where, and how does the spatial heterogeneity of soils matter? Ecol Model, 2015;298:2438.

[8] Baveye, P.C., 2015. Grand challenges in the research on soil processes. Front Environ Sci, 2015;3,10.

[9] Blunt M.J., Bijeljic B., Dong H., Gharbi O., Iglauer S., Mostaghimi P., Paluszny A., Pentland C. Pore-scale imaging and modelling. Adv Water Resour, 2013;51:197-216.

[10] Brusseau M.L., Peng S., Snaar G., Murao A. Measuring Air-Water Interfacial Areas with X-ray Microtomography and Interfacial Partitioning Tracer Tests. Environ Sci Technol, 2007;41:1956-1961.

[11] Carminati A., Kaestner A., Lehmann P., Flühler H. Unsaturated water flow across soil aggregate contacts. Adv Water Resour, 2008;31:1221-1232.

[12] Culligan K.A., Wildenschild D., Christensen B.S.B., Gary W.G., Rivers M.L. Pore-scale characteristics of multiphase flow in porous media: A comparison of air-water and oil-water experiments. Adv Water Resour, 2006;29:227-238.

[13] Falconer R.E., Houston A.N., Otten W., Baveye P.C. Emergent Behavior of Soil Fungal Dynamics: Influence of Soil Architecture and Water Distribution. Soil Sci, 2012;177:111-119.

[14] FA0, 2006. World Reference Base for Soil Resources 2006. A Framework for International Classification, Correlation and Communication. World Soil Resources Reports 103, 145pp. Food and Agriculture organization of the United Nations, Rome.

[15] Folse III H.J., Allison S.D. Cooperation, competition, and coalitions in enzyme-producing microbes: social evolution and nutrient depolymerization rates. Front Microbiol, 2012;3,338.

[16] Fraunhofer ITWM. Department of Image Processing. ToolIP - tool for image processing. http://www.itwm.fraunhofer.de/toolip, 2009.

[17] Fraunhofer ITWM. Department of Image Processing. MAVIkit MAVI toolbox for ToolIP. http://www.itwm.fraunhofer.de/toolip, 2013. 
[18] Garnier P., Cambier C., Bousso M., Masse D., Chenu C., Recous S. Modeling the influence of soil-plant residue contact on carbon mineralization: Comparison of a compartmental approach and a 3D spatial approach. Soil Biol Biochem, 2008;40:2754-2761.

[19] Gawin D., Sanavia L. Simulation of cavitation in water saturated porous media considering effects of dissolved air. Transport Porous Med, 2010;81:141-160.

[20] Genty A., Pot V. Numerical simulation of 3D liquid-gas distribution in porous media by a two-phase TRT lattice Boltzmann method. Transp Porous Med, 2013;96:271-294.

[21] Genty A., Pot V. Numerical calculation of effective diffusion in unsaturated porous media by the TRT lattice Boltzmann method. Transp Porous Med, 2014;105:391-410.

[22] Ginzburg, I., Verhaeghe, F., d'Humières, D. Study of simple hydrodynamics solutions with the two-relaxation-times lattice Boltzmann scheme. Commun Comput Phys, 2008;3:519-581.

[23] Hapca S.M., Houston A., Otten W., Baveye P.C. New local thresholding method for soil images by minimizing grayscale intra-class variance. Vadose Zone J, 2013;12(3):doi:10.2136/vzj2012.0172.

[24] Houston, A.N., Otten, W., Baveye, P.C., Hapca, S.M. Thresholding of computed tomography images of heterogeneous porous media by adaptive-window indicator kriging. Comput Geosci 2013;54:239248.

[25] Houston A.N., Schmidt S., Tarquis A.M., Otten W., Baveye P.C., Hapca S.M. Effect of scanning and image reconstruction settings in X-ray computed microtomography on quality and segmentation of 3D soil images. Geoderma, 2013;207-208(1):154-165.

[26] Hunt A.G., Ewing R.P., Horton R. What's Wrong with Soil Physics? Soil Sci Soc Am J, 2013;77:1877-1887.

[27] Knutson C.E., Werth C.J., Valocchi A.J. Pore-scale simulation of biomass growth along the transverse mixing zone of a model two-dimensional porous medium. Water Resour Res, 2005;41,W07007.

[28] Luxmoore R. Micro-, meso- and macroporosity of soil. Soil Sci Soc Am J, 1981;45:671-672.

[29] Martys, N.S., Chen, H. Simulation of multicomponent fluids in complex three-dimensional geometries by the lattice Boltzmann method. Phys Rev E, 1996;53(1):743-750.

[30] Masse D., Cambier C., Brauman A., Sall S, Assigbetse K., Chotte J.-L. MIOR: an individual-based model for simulating the spatial patterns of soil organic matter microbial decomposition. Eur J Soil Sci, 2007;58:1127-1135.

[31] Miller E.E., Salehzadeh A. Stripper for bubble-free tensiometry. Soil Sci Soc Am J, 1993;57:1470-1473.

[32] Miller R.D. Comment on "Paradoxes and realities in unsaturated flow theory" by W.G. Gray and S.M. Hassanizadeh. Water Resour Res, 1994;30:1623-1624.

[33] Monga, O., Ngom, N.F., Delerue, J.F. Representing geometric structures in 3D tomography soil images: Application to pore space modelling. Comput Geosci, 2007;33:1140-1161.

[34] Monga, O., Bousso, M., Garnier, P., Pot, V. 3D geometric structures and biological activity: Application to microbial soil organic matter decomposition in pore space. Ecol Modell, 2008;216:291-302.

[35] Monga O., Garnier P., Pot V., Coucheney E., Nunan N., Otten W., Chenu C. Simulating microbial degradation of organic matter in a simple porous system using the 3-D diffusion-based model MOSAIC. Biogeosciences, 2014, 11:2201-2209.

[36] Ngom N.F., Monga O., Mohamed M.M.O., Garnier P. 3D shape extraction segmentation and representation of soil microstructures using generalized cylinders. Comput Geosci, 2012;39:50-63.

[37] Ollion J., Cochennec J., Loll F., Escudé C., Boudier T. TANGO: A Generic Tool for High-throughput 3D Image Analysis for Studying Nuclear Organization. Bioinformatics, 2013:29:1840-1841

[38] Or D., Tuller M. Cavitation during desaturation of porous media under tension. Water Resour Res, $2002 ; 38,1061$.

[39] Otsu N. A threshold selection method from gray level histograms. EEE Trans Systems, Man and Cybernetics, 1979;9:6266.

[40] Peth S., Horn R., Beckmann F., Donath T., Fisher J., Smucker A.J.M. Three-Dimensional Quantification of Intra-Aggregate Pore-Space Features using Synchrotron-Radiation-Based Microtomography. Soil Sci Soc Am J, 2008;72:897-907.

[41] Peth S., Nellesen J., FischerG., Horn R. Non-invasive 3D analysis of local soil deformation under mechanical and hydraulic stresses by $\mu$ CT and digital image correlation. Soil Till Res, 2010;111:3-18.

[42] Raiskinmäki, P., Koponen, A., Merikoski, J., Timonen, J. Spreading dynamics of three-dimensional droplets by the lattice Boltzmann method. Comp Mater Sci, 2000;18:7-12.

[43] Schaap M.G., Porter M.L., Christensen B.S.B., Wildenschild D. Comparison of pressure-saturation characteristics derived from computed tomography and lattice Boltzmann simulations. Water Resour Res, 2007;43:W12506.

[44] Shan, X., Chen, H. Lattice Boltzmann model for simulating flows with multiple phases and components. Phys Rev E, 1993;47(3):1815-1820.

[45] Shan, X., Chen, H. Simulation of nonideal gases and liquid-gas phase transitions by the lattice Boltzmann equation. Phys Rev E, 1994;49(4):2941-294.

[46] Sukop M.C., Huang H., Lin C.L., Deo M.D., Oh K., Miller J.D. Distribution of multiphase fluids in porous media: Comparison between lattice Boltzmann modeling and micro-X-ray tomography. Phys Rev E, 2008;77:026710.

[47] Tippkötter R., Eickhorst T., Taubner H., Gredner B., Rademaker G. Detection of soil water in macropores of 
undisturbed soil using microfocus X-ray tube computerized tomography $(\mu \mathrm{CT})$. Soil Till Res, 2009;105:12-20.

[48] Taina I.A., Heck R.J., Elliot T.R. Application of X-ray computed tomography to soil science: A literature review. Can J Soil Sci, 2008;88:1-20.

[49] Van Loo D., Bouckaert L., Leroux O., Pauwels E., Dierick M., Van Hoorebeke L., Cnudde V., De Neve S., Sleutel S. Contrast agents for soil investigation with X-ray computed tomography. Geoderma, 2014;213:485-491.

[50] Vogel H.-J., Roth K.I. Quantitative morphology and network representation of soil pore structure. Adv Water Res, 2001;24:233-242.

[51] Vogel H.-J., Tölke J., Schulz V.P., Krafczyk M., Roth K. Comparison of a Lattice-Boltzmann Model, a FullMorphology Model, and a Pore Network Model for Determining Capillary Pressure-Saturation Relationships. Vadose Zone J, 2005;4:380-388.

[52] Vogel H.-J., Weller U., Schlüter S. Quantification of soil structure based on Minkowski functions. Comput Geosci, 2010;36:1326-1245

[53] Vogel L., Makowski D., Garnier P., Vieublé-Gonod L., Raynaud X., Nunan N., Coquet Y., Chenu C., Pot V. Modelling the effect of soil meso- and macropores topology on the biodegration of a soluble carbon substrate. Adv Water Resour, 2015;83:123-136. 